\title{
Childhood Disorders and Developmental Influences: A 10-Year Content Analysis of Two Prominent Journals
}

\author{
Bridget A. Walsh ${ }^{*}$, , Amy R. Murrell ${ }^{\mathrm{b}}$, Andrew J. Scherbarth ${ }^{\mathrm{b}}$ and Chelsea Rae Kubiak ${ }^{\mathrm{b}}$ \\ ${ }^{a}$ Department of Family Sciences, Texas Woman's University, Denton, TX, USA \\ ${ }^{b}$ Department of Psychology, University of North Texas, Denton TX, USA
}

\begin{abstract}
Many scholars and practitioners prefer to use a developmental approach toward investigation and treatment of child psychopathology. However, the extent to which development is considered in childhood disorder research was unclear. Therefore, retrospective analyses were conducted of publications from 1996 to 2005 in a prominent abnormal child psychology journal (Journal of Abnormal Child Psychology; $\mathrm{N}=472$ ) and a prominent developmental psychology journal (Developmental Psychology; $\mathrm{N}=926$ ), to investigate the frequency of appearance of developmental factors and childhood disorders. Data on author affiliation and type, and acknowledgement of funding, were also analyzed. Our findings were consistent with a previous analysis; most studies were conducted by funded, university-affiliated researchers. Some disorders, including those typically construed as developmental in nature, (e.g., PDD-NOS) appeared significantly more in the abnormal than the developmental journal. Pathology was infrequently mentioned in the journal with a developmental focus. Implications of these findings are discussed. It is reasonable to suggest that the present analysis may lead to important changes in policy and resource allocation as relevant to children and their families.
\end{abstract}

\section{INTRODUCTION}

In 2005 a prominent abnormal child psychology journal included a special issue of empirical works and a commentary heralded by Salekin and Frick's [1] introduction that focused on the "need for a developmental perspective" in studying child and adolescent psychopathology. Prior to this issue, other publications in this abnormal child psychology journal have had a developmental focus [2-9]. There is wide agreement that psychological problems should be investigated from a developmental perspective according to Lahey, Miller, Schwab-Stone et al. [10], yet the extent to which this perspective is actually utilized is unclear. In order to answer that question, these authors conducted a content analysis of recent child clinical research. More specifically, the extent to which development was addressed in child psychopathology research was examined.

A developmental approach allows for focus on adaptive functioning as well as problems, and it seems reasonable that this dual focus better represents the entirety of a child and adolescent's experience as well as provides information about the origins and courses of various disorders [11]. There are a variety of characteristics that have been used to describe a developmental perspective; however, all descriptions include the following assumptions: (1) human development is dynamic and yet predictable, (2) person and environment are interactive and, thus, context is important which is consistent with studying family factors, and (3) normal processes are relevant to deviations, including psychopathology, especially in terms of timing and degree.

*Address correspondence to this author at the Family Sciences, Texas Woman's University, P.O. Box 425769, Denton, TX, 76204-5769, USA;

E-mail: bwalsh1@twu.edu
The link between normal processes and psychopathology is described within developmental psychopathology. Developmental psychopathology is concerned with the precursors and risk factors that are relevant to development and maintenance of disorders and how such disorders are manifest in varying ways across time, as well as with how resulting problems are related to normal development [12]. This approach calls for an examination of factors including: chronological and mental age, gender, physical and hormonal maturation, level of social functioning, family factors, and life experiences [13]. This approach has been in favor since the 1980s [14].

Since then, several psychologists [11-13] have noted the implications of considering development in the assessment, diagnosis, and treatment of child and adolescent psychopathology. For example, knowledge of children's agerelated cognitive and motor functioning is crucial for choosing appropriate assessment measures. Young children may have difficulties in the reading and monitoring that is required to complete self-report measures. Even more basic, they may not yet be able to sit, focus, and use a writing utensil. Thus, parent and teacher reports, or behavioral observations, are more effective assessment tools when working with young children. However, a clinician would certainly want to ask an adolescent to report on his or her own behavior. Parents and teachers are often not aware of problematic behaviors or symptoms that are experienced by older children and adolescents. In addition, children and adolescents at this age could be very reactive to observation [11].

Having an awareness of typical development also helps to define what is considered deviant behavior; and, therefore, assists in the identification of what should be addressed in case conceptualization and treatment planning. According to 
Garber [15], a developmental perspective to child psychopathology allows for the differentiation of normality and deviance by adding the assessment of contextual factors, such as age-related abilities and behaviors. For example, elimination problems are common in early childhood but quite rare in adolescence. A 2-year-old who "has accidents" is probably far less distressing to others than an 11-year-old who does. Aggression and refusal to share in young children, who cannot yet take another's viewpoint, are likely related to cognitive abilities rather than psychopathology [14]. In fact, many toddlers commonly have these "problems." Aggressive behaviors are more the norm than deviation until higher level processing develops. More generally, Garber [15] notes that data collected in non-clinical samples suggests that behavioral problems are common, especially among boys, in early childhood and appear to decline as children age.

It is important to note that childhood psychopathology may present differently than adult difficulties. For example, children with Obsessive-Compulsive Disorder do not necessarily recognize the irrationality of their thoughts. However, this recognition is a key part of the diagnosis in adulthood. Related to that, depressed children do not worry about the future in the same way that depressed adults worry about it. In the diagnosis of mood disorders there are, in fact, several age-related diagnostic differences concerning duration and quality of symptoms.

Given varying presentation, it is important to examine indirect factors related to case presentation and prognosis. The developmental approach lends itself to the investigation of indirect effects. It is well-known, for example, that gender mediates and/or moderates a variety of outcomes. Holmbeck, Abad, Friedman, and Jandasek [16] in a recent review chapter note that early maturation in girls leads to several negative outcomes that are not noted in early maturing boys. Disregard for indirect factors, as well as for developmental factors more generally, can lead to over- or underpathologizing and less effective treatment. Consider that it would likely be an inefficient use of time and energy to work with a 2-year-old on seeing her mother's perspective when she tantrums, although teaching empathy skills to a defiant adolescent might be highly effective. It is crucial, therefore, to consider age and developmental abilities when diagnosing and treating. Typically, the earlier that problems develop, the more problems that are experienced, and the longer that such problems persist, the worse the prognosis [14]. However, this is not always the case. For reasons similar to this diversity in outcome, it is important to consider process and individual differences in psychopathology research within a developmental paradigm [13].

\section{DEVELOPMENTAL PARADIGM AND CONTENT ANALYSIS}

This purpose of the present content analysis is twofold. First, such research is a reflective step for journal editors and researchers who wish to consider publication practices and trends. Second, results of content analyses can be used to define the discipline [17]. Findings that would give journal editors, researchers, and readership interested in child pathology and related developmental issues the most insight about who conducted previous research as well as what variables are relevant to previous research type and topic were chosen based on a previous content analysis published in a similar area. Specifically, Wasserstein, Lopez, and Routh's [18] review of the Journal of Abnormal Child Psychology (JACP) from 1973 to 1996 included in its investigation: (1) contributors' type of institutional affiliation; (2) geographical location; and (3) if contributors acknowledged research funding. The current analysis examined various trends and changes in the three aforementioned dimensions established in Wasserstein, Lopez, and Routh's [18] review. In addition, a tally of the number of authors for each publication was performed in order to get a sense of collaboration evidenced in these publications and to help further determine who is conducting the research. Prior to the present study, Wasserstein, Lopez, and Routh's [18] work was the only content analysis of publications in the selected abnormal child psychology journal.

Wasserstein, Lopez, and Routh [18] reported that most first authors were affiliated with universities $(70 \%)$, and that publications from hospitals and medical schools increased with time. This finding suggests that a content analysis starting with publications in 1996 may reveal a continued increase of publications with first authors affiliated with hospitals and medical schools.

Most publications (83\% of 960 publications) in the Wasserstein, Lopez, and Routh's [18] review had first authors affiliated with institutions in the United States. First authors with a primary institutional affiliation outside of the U.S. increased with time. This is a revealing finding in view of the fact that the JACP has a national and international scope in authorship and readership. Wasserstein, Lopez and Roth [18] indicated that, at the time of their publication, editorial board members were then being selected from institutions all over the world, further supporting that first authors outside of the U.S. would be expected to increase with time. Moreover, these researchers proposed that this internationalization was most likely true of research in the child and adolescent psychopathology field. All in all, this suggests that a more recent analysis may indicate a more equal percentage of publications from first authors affiliated with American and non-American institutions. In Wasserstein, Lopez, and Routh's [18] review, approximately half of the publications reviewed were the result of funding. During the years that the reviewed articles were published, the number of funded projects increased little. This finding suggests that a robust increase in publications reporting funding in the JACP is not probable.

\section{PRESENT STUDY}

As previously stated, the analysis conducted for this study examined institutional affiliation, geographical location, funding, and number of authors for a number of child related publications. This analysis was performed in order to get a sense of collaboration evidenced in these publications and to help further determine who is conducting such research. To do these analyses, articles published from 1996 to 2005 in a prominent abnormal child psychology journal (Journal of Abnormal Child Psychology; JACP) and in a prominent developmental psychology journal were reviewed (Developmental Psychology; DP). Wasserstein, Lopez, and Routh's [18] content analysis was limited to one journal, JACP. While this journal may include projects that 
address developmental factors, it does not specifically use a developmental framework. Therefore, the authors did not examine content from a developmental perspective or compare information from an abnormal child psychology journal and a developmental psychology journal. Consequently, the present study is an advancement to the extant literature.

The current content analysis allowed for investigation of whether the frequency of the appearance of the major childhood disorders was any higher in a prominent abnormal child psychology journal than in a prototypical developmental psychology journal. It was expected that the abnormal psychology journal would give more coverage of child psychopathology. In addition, this project served to determine our other specific interest-whether publications in these two journals directly acknowledged a developmental perspective with this approach emphasized more in the developmental journal. Stated differently, we examined the extent to which research from an abnormal child psychology journal and a developmental journal addressed developmental factors. We expected that a journal that underscores psychopathology but that has previously acknowledged the importance of a developmental framework would have a high representation of articles that consider developmental factors, but that such representation would yet be higher in a developmental journal. To reiterate, we also expected a similar percentage of publications from first authors affiliated with American and non-American institutions in the Journal of Abnormal Child Psychology, with the latter increasing over time.

\section{METHOD}

\section{Selected Journals}

Developmental Psychology (DP) is a highly respected American Psychological Association (APA) journal. Articles in DP emphasize human development, and articles focusing on specific aspects of development, such as psychological development, are also published [19]. This selected journal was among the top two most cited journals in the subfield of developmental psychology [20].

The journal selected to represent clinical psychology was the Journal of Abnormal Child Psychology (JACP), a journal of the International Society for Research in Child Development and Adolescent Psychopathology (ISRCAP). The JACP was somewhat modeled after the Journal of Abnormal Psychology [18], which had the same placement on rankings and ratings in the clinical subfield category as DP did in the developmental category [20]. The JACP focuses on the major childhood disorders as well as other dimensions, such as assessment and treatment, and considers the developmental course of childhood and adolescent disorders [21]. Given the aim of the present study, the JACP's emphasis on major childhood disorders and developmental trajectory prompted our selection of this journal.

\section{Data Sources and Instrument}

Several electronic resources were utilized. Two university library electronic database systems were used to view the full text of publications. The prominent abnormal child psychology journal was accessed via two online databases: SpringerLink and ProQuest Social Science Journals, Wilson Social Sciences Abstracts. The prominent developmental psychology journal was accessed and viewed via one online database: PsycInfo. All searched publications were opened in Portable Document Format (PDF) via Adobe Reader version 7.0. Moreover, SPSS version 12.0 was used to process all statistics. Microsoft Excel was used for the statistical check of reliability.

Each full publication within the target journal was viewed and electronically searched for the target words stated in the procedure. A data sheet was utilized for each publication and organized the information about each journal's annual activity. Type of institutional affiliation (i.e., hospital, medical school, university, or other) and geographic location (i.e., inside or outside of the U.S.) of each publication's first author was noted. Explicit mention of funding was coded. To advance the content analysis literature, direct acknowledgement of a developmental perspective and searched childhood disorders were also tracked. Finally, number of authors for each publication was coded, thus expanding upon Wasserstein, Lopez, and Routh's [18] review of author information.

\section{Procedure}

The analyzed publications were found in the Journal of Abnormal Child Psychology (JACP) and Developmental Psychology (DP), published from 1996 to 2005. Publications $(\mathrm{N}=472)$ were investigated in this prominent abnormal child psychology journal (hereafter referred to as JACP) over this 10 year time span, thus including Volumes 24-33. Additionally, publications $(\mathrm{N}=926)$ were investigated in this prominent developmental psychology journal (hereafter referred to as DP) from the same aforementioned decade, thus including Volumes 32-41. Titles and entirety of texts were separately searched for references to childhood disorders and/or developmental perspective.

The search words for child psychopathology in the text and title of each publication included: Disruptive Behavior Disorder(s) (DBDs), Conduct Disorder (CD), Oppositional Defiant Disorder (ODD), Attention Deficit Hyperactivity Disorder (ADHD), Anxiety Disorder(s), anxiety symptom(s), Depressive Disorder(s), depressive symptom(s), depression, and the Pervasive Developmental Disorder(s) (PDDs). Given the developmental focus of this analysis we also searched for types of Pervasive Developmental Disorders, these were: Autistic Disorder or Autism, Rett's Disorder, Childhood Disintegrative Disorder, Asperger's Disorder, and Pervasive Developmental Disorder-Not Otherwise Specified (PDD-NOS).

An important issue is that neither anxiety symptoms nor depressive symptoms were searched for in the context of being a diagnosis. These two terms were viewed as general terms that could be due to multiple causes, as according to the fourth edition of the Diagnostic and Statistical Manual of Mental Disorders (DSM-IV-TR) [22] anxiety symptoms and depressive symptoms contribute to the diagnosis of multiple disorders. Therefore, given comorbidity, individual publications may contain more than one search word; all search words for each publication were coded to give an accurate representation of the typical hit rate for words related to child psychopathology. 
A search was conducted to find reference to inclusion of a developmental perspective within the title and text of each publication. The search word, develop, served to provide information about the frequency of the explicit acknowledgment of a developmental perspective (e.g., developmental trends, developmental factors, stages of development, etc.). In general, search words that appeared in the references, announcements or editorial comments of each journal, regardless of the context, were excluded from analyses. Again, publications often contained key search words multiple times. For example, one publication that contained the phrase "developmentally appropriate" four times and another publication that featured it twice were each counted as one develop hit or tally. The more overall hits or tallies per search word across publications, the greater the frequency of the appearance of the developmental perspective and/or childhood disorders in the journal.

\section{Statistical Procedures}

All data received were entered into SPSS for analysis. For inferential statistics, annual percentage scores were used rather than raw scores in order to reduce error variance caused by the disproportionately larger number of publications printed annually in the $D P(M=92.60, S D=$ $21.13)$ than the $J A C P(M=47.10, S D=4.91), t(18)=-6.63$, $p<.001$. Two-tailed significance tests were used for all analyses. Type of institutional affiliation was compared using a two-way ANOVA that examined main and interaction effects of journal title and institution type. Independent-samples t-tests were conducted to compare differences on variables between journals as well as look at differences among variables within each journal. Comparisons were based on annual rate of author's geographic location, grant or funding acknowledgement, and developmental perspective acknowledged in the publication text. In addition, a series of independent-samples t-tests were used to compare rate of publications that incorporated specific child psychopathology words. An alpha level of .001 was utilized to determine significant relationships between the two journals in order to control for chance results due to the use of multiple t-tests. All statistical probabilities were computed using a two-tailed probability calculation method.

\section{Reliability}

Reliability analyses were conducted to assure integrity of data collection. A coauthor repeated the aforementioned search process on a randomly selected $20 \%$ of publications in the DP. The coauthor independently coded 185 publications from the DP using the coding procedure that was employed for the total sample as described in the methodology. Additionally, a research assistant of the second author independently coded 95 publications (i.e., $20 \%$ ) that were randomly selected from the JACP and applied the same coding framework. The coauthor and interrater totaled the data for each year of the 10 years under scrutiny. This data was then entered into an Excel database. Discrepancies in data, such as publication omissions, were then coded and added to the Excel database. Simple percentage agreements were calculated between the original data and the coauthor and inter-rater's data. To finalize, decimals were rounded to two places then converted to percentages.

\section{RESULTS}

\section{Reliability}

This inter-rater reliability procedure evidenced a score of 99\% agreement for first author information (type of institutional affiliation, geographic location, funding, and authorship) for the DP. Additionally for the DP, an agreement score of $97 \%$ was attained for the agreement of the childhood disorder search, and a score of $98 \%$ for the agreement of the acknowledgment of a developmental perspective. The inter-rater reliability for the JACP was: $97 \%$ for first author information; $93 \%$ for childhood disorders; and $91 \%$ for acknowledgment of a developmental perspective.

\section{Number of Publications}

The number of publications per volume increased over 10 years in the JACP, $r(10)=.82, p<.01$. Alternatively, the number of publications per volume decreased over a decade in the DP, $r(10)=-.57, p>.05$.

\section{Authorship and Affiliation}

Type of institutional affiliation. A 2 x 4 ANOVA was run to test the main and interaction effects of the two-levels of journal titles (i.e., JACP and DP) and four-levels of first author institutional affiliation (i.e., hospital, medical school, university and other), with percentage of publications per year as the dependent variable. There was no significant main effect for journal title, $F(1,72)=.00, p>.05$, partial eta-squared $\left(\eta_{\mathrm{p}}^{2}\right)=.00$. However, there was a significant main effect found for institutional affiliation, $F(3,72)=$ $1882.57, p>.001, \eta_{\mathrm{p}}{ }^{2}=.98$, such that university affiliation was the most commonly associated institution by a profound margin for all publications combined. A significant interaction effect was also found, $F(3,72)=33.20, p>.001$, $\eta_{\mathrm{p}}{ }^{2}=.58$. Results of independent pairwise comparisons conducted to analyze simple interaction effects are listed in Table 1.

Table 1. Simple Interaction Effects: Independent Pairwise Comparisons for Journal Title and Institutional

\begin{tabular}{|c|c|c|c|c|}
\hline Affiliation Interaction & df & $\mathbf{F}$ & $\mathbf{P}$ & $\boldsymbol{\eta}_{\mathrm{p}}^{2}$ \\
\hline \multicolumn{5}{|c|}{ Journal Title by Institutional Affiliation } \\
\hline $\mathrm{JACP}^{1} \mathrm{x}$ Affiliation Type & 3 & 730.31 & $<.001$ & .97 \\
\hline $\mathrm{DP}^{2} \mathrm{x}$ Affiliation Type & 3 & 1185.46 & $<.001$ & .98 \\
\hline Error & 72 & & & \\
\hline \multicolumn{5}{|c|}{ Institutional Affiliation by Journal Title } \\
\hline Hospital x Journal & 1 & 2.41 & $>.05$ & .03 \\
\hline Medical School x Journal & 1 & 33.24 & $<.001$ & .32 \\
\hline University x Journal & 1 & 63.51 & $<.001$ & .47 \\
\hline Other x Journal & 1 & .42 & $>.05$ & .01 \\
\hline Error & 72 & & & \\
\hline
\end{tabular}

Comparisons of percentage of publications with each first author's institutional affiliation within each journal reveal 


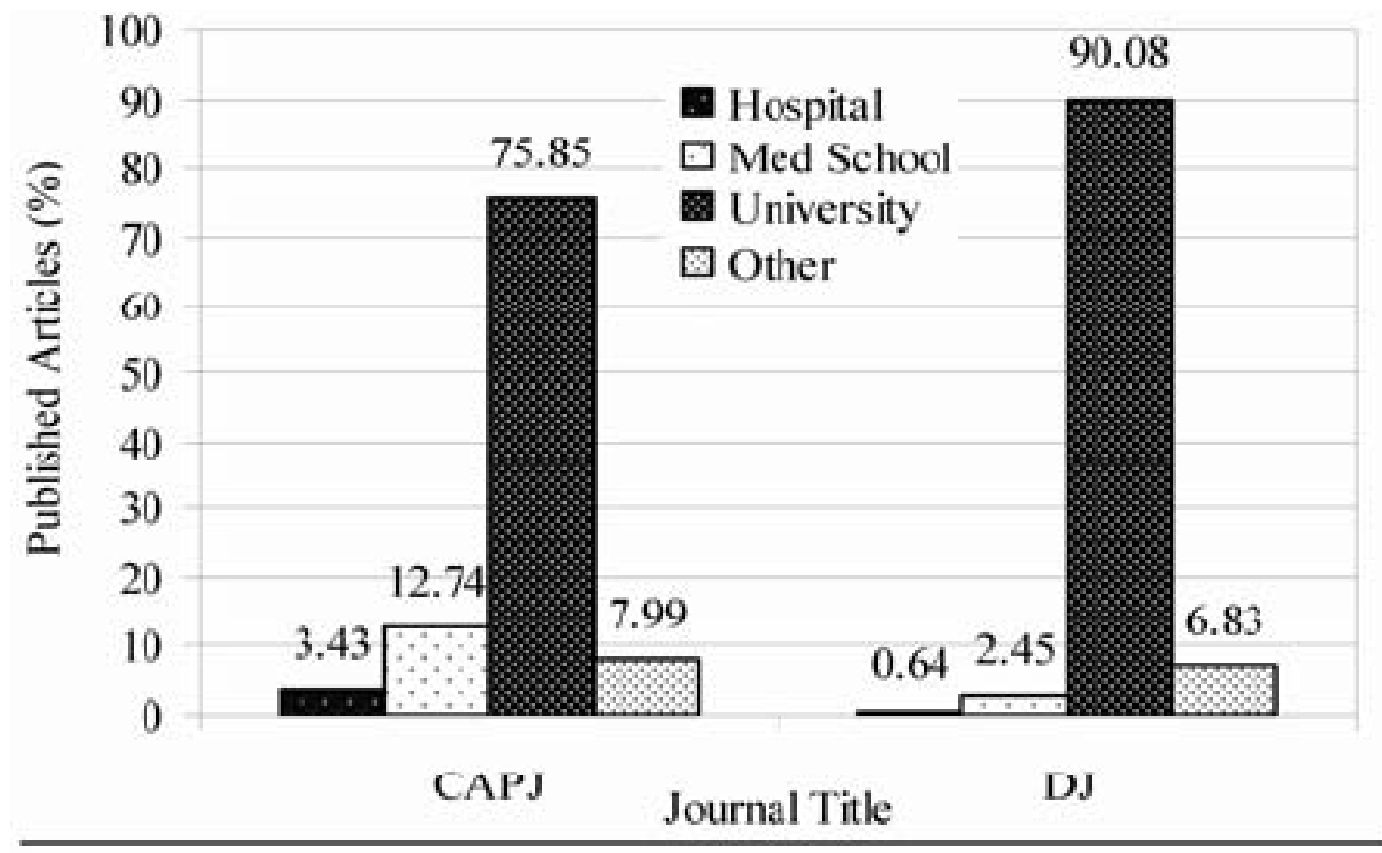

Note. JACP $=$ Journal of Abnormal Child Psychology, DP $=$ Developmental Psychology.

Fig. (1). Bar graph of simple interaction effect: Journal title by institutional affiliation.

significant group differences. In the JACP, university affiliation was most common, followed by medical school, other affiliation and hospital affiliation. In the DP, university affiliation was also the most common, followed by other institutional affiliation. Hospital and medical school submissions were non-significantly different from one another and published least frequently. Graphical representation of these analyses are presented in Fig. (1).

Overall, first author affiliations with universities (76\% JACP and $90 \%$ DP) were the most frequent. This was compared to $13 \%$ of first authors affiliated with a medical school in the JACP and $2 \%$ in the DP. Also, 3\% of first authors affiliated with a hospital in the JACP and less than $1 \%$ in the DP. Finally, $8 \%$ of first authors were affiliated with another type of institution (e.g., the National Institute of Health in the United States) in the JACP and 7\% (e.g., Max Planck Institute) in the DP.

Geographic location. A significant majority of first authors reported more frequent affiliation with an institution in the United States as compared to an institution abroad. This held true for both the JACP $(M=74.82 \%, S D=7.06)$, $t(18)=15.72, p<.001$, and the DP $(M=70.21 \%, S D=$ $4.78), t(18)=18.92, p<.001$. By contrast, the publication rate for authors with an affiliation inside of the U.S. is not statistically different between journals, $t(18)=1.71, p>.05$.

Number of authors. The majority of publications were written by single authors or groups of 2-4 authors. In the JACP, three authors was the number of authors most featured $(24 \%)$. While $23 \%$ of the publications had two authors and $21 \%$ of the publications featured four authors. In the DP, two authors was the most prevalent (31\%), whereas three authors had the second highest total (25\%). Approximately $1 \%$ of the total publications in each of the investigated journals listed a research group, network or study group in the authorship. See Fig. (2) for comparisons.

\section{Funding}

In the JACP, significantly more publications reported funding $(M=69.68 \%, S D=4.89)$, than did those who did not report funding $(M=30.32, S D=4.89), t(18)=18.00, p<$ .001 . The same held true for the DP, which reported an average of $84.51 \%(S D=7.03)$ publications that reported funding, as opposed to those who did not $(M=15.49 \%, S D=$ 7.03), $t(18)=21.94, p<.001$. Overall, significantly more studies reported funding in DP than in the JACP, $t(18)=$ $5.47,<.001$.

\section{Childhood Disorders}

The frequency of childhood disorders stated in each journal was compared. Analyses demonstrated a higher rate of publications with $\mathrm{CD}, \mathrm{ADHD}$, depression and depressive symptoms in the article title in the abnormal child psychology journal compared to the developmental journal. Additionally, the hit rate for DBD, CD, ODD, ADHD, Anxiety Disorders, anxiety symptoms, depression, Depressive Disorder, depressive symptoms, PDD and PDDNOS were all significantly higher in the text of the JACP than the DP. Tables $\mathbf{2}$ and $\mathbf{3}$ include annual frequency of publication and mean comparison rates between the two journals.

Childhood psychopathology word hit rates varied according to journal and whether they were listed in the title or text. For the JACP, ADHD was included in nearly $20 \%$ of publication titles, while rates of other major childhood disorders ranged from $0.5-5 \%$. A majority of publications mentioned depression in the text, while hit rates for disruptive behavior disorders were in the $40 \%$ range and anxiety disorders were mentioned in nearly one-third of publications in text. By comparison, article titles in the DP 


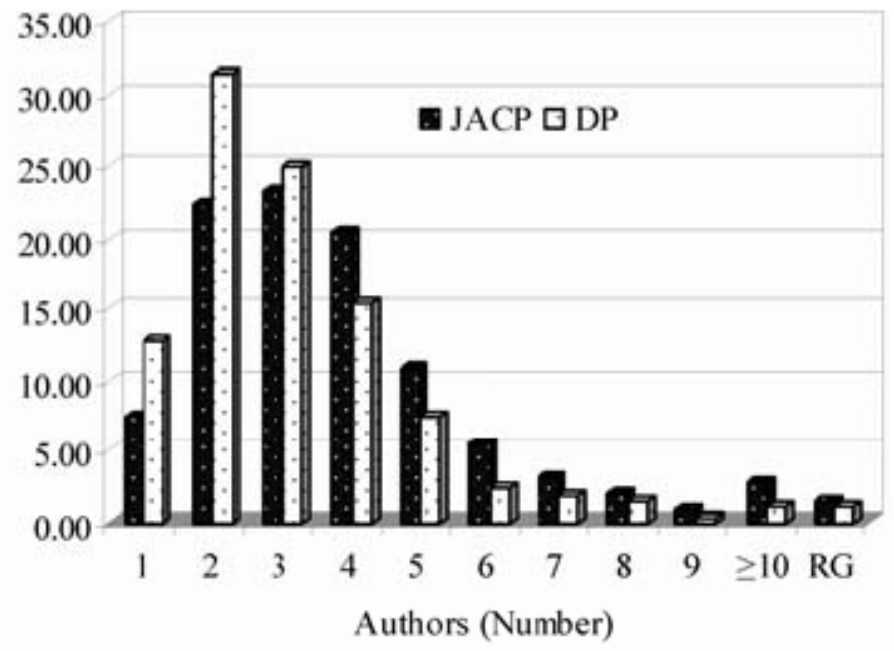

Note. JACP $=$ Journal of Abnormal Child Psychology, DP = Developmental Psychology $\mathrm{RG}=$ Research Group.

Fig. (2). Percentage of publications by number of authors from JACP $(\mathrm{N}=472)$ and DP $(\mathrm{N}=928)$.

Table 2. Title Rate of Publications Annually that Include Typical Childhood Disorders, 1996-2005

\begin{tabular}{|c|c|c|c|c|c|}
\hline \multirow{2}{*}{ Search Words } & \multicolumn{5}{|c|}{ Title } \\
\cline { 2 - 6 } & \multicolumn{2}{|c|}{ JACP $^{1}$} & \multicolumn{3}{c|}{ DP $^{2}$} \\
\cline { 2 - 6 } & M & SD & M & SD & t \\
\hline \hline DBD $^{3}$ & .67 & $(1.08)$ & .00 & $(.00)$ & 1.96 \\
\hline Conduct Disorder $^{*} 2.66$ & $(2.05)$ & .22 & $(.46)$ & $3.69 \dagger$ \\
\hline ODD $^{6}$ & 3.11 & $(3.87)$ & .00 & $(.00)$ & 3.01 \\
\hline ADHD $^{*}$ & 19.06 & $(5.31)$ & .18 & $(.39)$ & $11.21 \dagger$ \\
\hline Anxiety Disorder(s) & 1.86 & $(2.67)$ & .00 & $(.00)$ & 2.20 \\
\hline Anxiety Symptom(s) & .25 & $(.79)$ & .00 & $(.00)$ & 1.00 \\
\hline Depression & 4.84 & $(3.11)$ & .26 & $(.59)$ & $4.58 \dagger$ \\
\hline Depressive Disorder(s) & .18 & $(.56)$ & .00 & $(.00)$ & 1.00 \\
\hline Depressive Symptom(s) & 3.64 & $(1.96)$ & 1.10 & $(1.15)$ & $3.53 \dagger$ \\
\hline PDD $^{4}$ & .00 & $(.00)$ & .15 & $(.47)$ & -1.00 \\
\hline Autism/Autistic & .83 & $(1.08)$ & .72 & $(.95)$ & .22 \\
\hline Asperger's & .21 & $(.67)$ & .00 & $(.00)$ & 1.00 \\
\hline PDD-NOS & .00 & $(.00)$ & .00 & $(.00)$ & 1.00 \\
\hline
\end{tabular}

Note. ${ }^{1}$ Journal of Abnormal Child Psychology, ${ }^{2}$ Developmental Psychology, ${ }^{3}$ Disruptive Behavior Disorders, ${ }^{4}$ Pervasive Developmental Disorder, ${ }^{5}$ Pervasive Developmental Disorder-Not Otherwise Specified, ${ }^{6}$ Oppositional Defiant Disorder. ${ }^{\dagger}$ $\mathrm{p}<.001$ (two-tailed).

with child were rare; depression had the highest annual frequency and was found in approximately $1 \%$ of publications. Rate of psychopathology mentioned in text for the DP were much higher, with depression tallied in $22 \%$ of publications, disruptive behavior disorder hit rates ranging from $1-5 \%$, anxiety hit rates in the $2 \%$ range and autism or other PDD hit rates of less than $4 \%$ each.
Table 3. Text Rate of Publications Annually that Include Typical Childhood Disorders, 1996-2005

\begin{tabular}{|c|c|c|c|c|c|}
\hline \multirow{2}{*}{ Search Words } & \multicolumn{5}{|c|}{ Text } \\
\cline { 2 - 6 } & \multicolumn{2}{|c|}{ JACP $^{1}$} & \multicolumn{3}{c|}{ DP $^{2}$} \\
\cline { 2 - 6 } & M & SD & M & SD & t \\
\hline \hline DBD $^{3}$ & 21.10 & $(7.13)$ & .90 & $(.96)$ & $8.87^{\dagger}$ \\
\hline Conduct Disorder $^{*}$ & 49.13 & $(6.77)$ & 5.16 & $(2.97)$ & $18.81^{\dagger}$ \\
\hline ODD $^{6}$ & 37.00 & $(7.05)$ & 1.56 & $(1.07)$ & $15.72^{\dagger}$ \\
\hline ADHD $^{*}$ & 46.16 & $(10.11)$ & 3.34 & $(1.97)$ & $13.13^{\dagger}$ \\
\hline Anxiety Disorder(s) & 30.12 & $(11.78)$ & 2.02 & $(1.26)$ & $7.50^{\dagger}$ \\
\hline Anxiety Symptom(s) & 17.29 & $(6.55)$ & 1.63 & $(1.19)$ & $7.43^{\dagger}$ \\
\hline Depression & 54.92 & $(4.45)$ & 22.48 & $(4.69)$ & $15.87^{\dagger}$ \\
\hline Depressive Disorder(s) & 16.70 & $(6.87)$ & 1.15 & $(.97)$ & $7.09^{\dagger}$ \\
\hline Depressive Symptom(s) & 23.57 & $(10.04)$ & 10.24 & $(3.82)$ & $3.93^{\dagger}$ \\
\hline PDD & 7.83 & $(4.30)$ & .10 & $(.32)$ & $5.67^{\dagger}$ \\
\hline Autism/Autistic & 6.75 & $(3.63)$ & 3.98 & $(2.63)$ & 1.96 \\
\hline Asperger's & .57 & $(1.25)$ & .41 & $(.96)$ & .33 \\
\hline PDD-NOS & .44 & $(.93)$ & .23 & $(.50)$ & $.62^{\dagger}$ \\
\hline
\end{tabular}

Note. ${ }^{1}$ Journal of Abnormal Child Psychology, ${ }^{2}$ Developmental Psychology, ${ }^{3}$ Disruptive Behavior Disorders, ${ }^{4}$ Pervasive Developmental Disorder, ${ }^{5}$ Pervasive Developmental Disorder-Not Otherwise Specified, ${ }^{6}$ Oppositional Defiant Disorder. ${ }^{\dagger}$ $\mathrm{p}<.001$ (two-tailed).

\section{Developmental Perspective}

Nearly three-quarters of the publications investigated in the JACP $(M=75.57, S D=8.07)$ included the search term development in an appropriate context in text (i.e., pertaining to child development only). A significantly large number of publications in the DP included a salient use of the word 
development $(M=96.50, S D=1.68)$. Therefore, the DP was more likely to contain publications that mention development compared to the JACP, $t(18)=8.03,<.001$. An informal observation was that most hits or tallies of this search word occurred in the introduction and/or discussion sections of the publication.

\section{DISCUSSION}

The present 10-year content analysis of publications permitted the examination of our results in comparison to a previous analysis of a prominent abnormal child psychology journal (i.e., JACP) conducted by Wasserstein, Lopez, and Routh [18]. Comparing our findings to those of Wasserstein, Lopez, and Routh [18], similarities exist, particularly in the dimensions of authorship and affiliation. These findings deserve attention because they point to important characteristics of the contributors in the fields of child psychopathology and developmental psychology.

Most first authors were affiliated with university departments (non-medical), consistent with Wasserstein, Lopez, and Routh's [18] finding. Albeit this was a robust trend in both investigated journals, this group is by no means the only producers of research in this sample. Medical schools, for example, are a fairly common place for research.

Wasserstein, Lopez, and Routh [18] found about half of the publications reported funding, and in our study funding was the rule rather than the exception. This was true in the JACP and DP and across all affiliations. While the present study reports on funding in general and did not distinguish between internal and external funding, there clearly has been a utilization of funding for developmental and/or child psychopathology issues as evidenced here. Though speculative, this finding may reflect that the need for consistent and long-term research programs in these broad areas is recognized.

We anticipated that the trend of first authors affiliated with institutions outside of the U.S. would be greater than $30 \%$ in the JACP based on Wasserstein, Lopez, and Routh's [18] investigation. Contrary to our expectation, international institutional affiliation did not increase over time. However, the number of authors outside of the U.S. seems to be approaching a percentage consistent with Wasserstein, Lopez, and Routh's [18] indication of the results from the first 24 Volumes of the JACP. Publications in general increased with time in the JACP, consistent with the trend reported by Wasserstein, Lopez, and Routh [18]. An observed trend worth noting is that first authors in the JACP and DP often collaborated with authors at different institutions and in different geographic locations outside of the country. The finding that most publications in both journals had two or three authors lends support to the idea about collaboration from different institutions.

The present study also provides an interesting and useful look at the similarities and differences between the frequency of coverage of major childhood disorders and/or a developmental perspective in research. The results of the content analysis of childhood disorders confirmed the expectation that the frequency of appearances for childhood disorders would be higher in the JACP compared to the DP. Specifically, the hit rate for $\mathrm{DBD}, \mathrm{CD}$, ODD, ADHD, Anxiety Disorders, anxiety symptoms, depression, Depressive Disorder, depressive symptoms, PDD and PDD-NOS were all significantly higher in the text of the JACP compared to the DP, suggesting that there is a need for developmentally focused journals to include more empirical work of child psychopathology while maintaining a developmental perspective. Given that the assessment, diagnoses, and treatment of children with childhood disorders, particularly PDDs, is best conducted by a multidisciplinary team of specialists that may incorporate child/adolescent psychiatrists, child psychologists, and developmental experts [23], studies that consider the link between normal processes and psychopathology serve as an important resource for practitioners.

There was convergence in the JACP and DP with respect to depression being the most frequently found search word. This high hit rate is congruent with Malmquist's [24] statement that depression is most likely the predominant affliction experienced by children. Additionally, our finding is supported by Costello, Erkanli, and Angold's [25] meta-analytic conclusion that high rates of depression in adolescents and children warrant reasonable concern. Another frequent search word occurrence was ADHD. Barkley [26] asserted that there are thousands of scientific papers focused on ADHD. Our finding that ADHD most frequently appeared in the titles of articles in the prominent abnormal child psychology journal partially supports Barkley's [26] statement.

Alternatively, PDDs, on average, had a low appearance or no appearance. In particular, PDD-NOS had a strikingly low hit rate in both investigated journals. Chakrabarti and Fombonne [27] found a precipitous increase in the last 15 years in the rate of reported PDDs with PDD-NOS as the most prevalent subtype in children. Consequently, it would be expected that the number of hit rates for PDDs would increase in a replication of the current content analysis in an examination of publications from 2005 to 2015 . The present work examined the bulwark of child research, which is an important source in decision making for resource allocation and education in schools and mental health clinics. If publications are under-representing increasing pathologies, such as PDD-NOS, then decision making in these settings will not have sufficient information to reach informed and effective decisions to help improve children's functioning.

Comorbidity of child and adolescent psychopathology [28] was not included in our frequency search. We acknowledge this as a limitation of our study. Moreover, excluding comorbidity as a search word in our analysis was not a reflection of extant publications in the two searched journals that may or may have not addressed this, rather it was not a specific interest in the current analysis. Nevertheless, the current findings are important as they call attention to knowledge about childhood psychological disorders and how they are portrayed, which enhances understanding of family life.

We were interested in determining the frequency of development being acknowledged in the JACP and DP. Indeed, our review indicated most publications explicitly stated developmental variables and perspectives. It is reasonable to conclude that our finding that almost three-quarters of searched publications in the JACP acknowledged a developmental perspective supports Campbell's [14] assertion that developmental psychopathology is a popular framework. The present study provides a foundation for future analyses to take an in-depth look at the concept of development by attending to the methodology in a prominent abnormal child psychology journal and prominent developmental journal. This study admittedly dichotomizes articles in terms of whether 
development was addressed based on limited search criteria. A closer look at development in journals via the investigation of the descriptions of samples in terms of gender, specific ages, and developmental periods may determine the precision in which sample compositions have been described, when applicable. Also, it will be important to determine if age-related functioning was specifically considered in the assessment, diagnosis, and treatment of child and adolescent psychopathology. A look at the contexts where the sample was recruited from (e.g., schools) and the research design employed (e.g., cross-sectional) may also contribute to understanding the developmental approach and illuminate directions for future research. Very importantly, a future content analysis that focuses on research design will determine the extent to which researchers controlled for developmental issues in their design and may or may not have made explicit reference to the concept of development.

Continued analysis of the construct of development in scholarly publications from abnormal child psychology focused journals and developmentally centered journals may encourage developmental concepts to be appropriately viewed in varied disciplines, such as family studies, and in broader literature. In turn, a clearer understanding of a developmental approach and factors in relation to psychopathology will continue to play a key role in researchers and practitioners informing and supporting interventions that improve the child's and adolescent's functioning. This is unquestionably important to understanding family functioning.

\section{ACKNOWLEDGMENT}

The authors would like to extend thanks to Jeffrey D. Geddes for assistance with the reliability procedure.

\section{REFERENCES}

[1] Salekin RT, Frick PJ. Psychopathy in children and adolescents: the need for a developmental perspective. J Abnorm Child Psychol 2005; 33: 403-9

[2] Ary DV, Duncan TE, Biglan A, Metzler CW, Noell JW, Smolkowski K. Development of adolescent problem behavior. J Abnorm Child Psychol 1999; 27: 141-51.

[3] Beyers JM, Loeber R. Untangling developmental relations between depressed mood and delinquency in male adolescents. J Abnorm Child Psychol 2003; 31: 247-66.

[4] Eyberg SM, Schuhmann EM, Rey J. Child and adolescent psychotherapy research: developmental issues. J Abnorm Child Psychol 1998; 26: 71-82.

[5] Gifford-Smith M, Dodge KA, Dishion TJ, McCord J. Peer influence in children and adolescents: Crossing the bridge from developmental to intervention science. J Abnorm Child Psychol 2005; 33: 255-65.

[6] Keenan K, Shaw D, Delliquadri E, Giovannelli J, Walsh B. Evidence for the continuity of early problem behaviors: Application of a developmental model. J Abnorm Child Psychol 1998; 26: 411-52.
[7] McCabe KM, Hough R, Wood PA, Yeh M. Childhood and adolescent onset conduct disorder: A test of the developmental taxonomy. J Abnorm Child Psychol 2001; 29: 305-16.

[8] McDermott PA. A nationwide study of developmental and gender prevalence for psychopathology in childhood and adolescence. $\mathrm{J}$ Abnorm Child Psychol 1996; 24: 53-66.

[9] Olson SL, Bates JE, Sandy JM, Lanthier R. Early developmental precursors of externalizing behavior in middle childhood and adolescence. J Abnorm Child Psychol 2000; 28: 119-33.

[10] Lahey BB, Miller TL, Schwab-Stone M, et al. Age and gender differences in oppositional behavior and conduct problems: a crosssectional household study of middle childhood and adolescence. J Abnorm Psychol 2000; 109: 488-503.

[11] Ollendick TH, Vasey MW. Developmental theory and the practice of clinical child psychology. J Clin Child Psychol 1999; 28: 45766.

[12] Sroufe LA, Rutter M. The domain of developmental psychopathology. Child Dev 1984; 55: 17-29.

[13] Rutter M. Multiple meanings of a developmental perspective on psychopathology. Eur J Dev Psychol 2005; 2: 221-52.

[14] Campbell SB. Developmental perspectives. In: Ollendick TH, Hersen M, Eds. Handbook of child psychopathology, $3^{\text {rd }}$ ed. New York: Plenum Press 1998; pp. 3-36.

[15] Garber J. Classification of childhood psychopathology: A developmental perspective. Child Dev 1984; 55: 30-48.

[16] Holmbeck GN, Abad M, Freidman D, Jandasek B. Development and psychopathology in adolescence. In: Wolfe DA, Mash EJ, Eds. Behavioral and emotional disorders in adolescence: Nature, assessment, and treatment. New York: Guilford 2006; pp. 21-55.

[17] Buboltz WC Jr, Miller M, Williams DJ. Content analysis of research in the Journal of Counseling Psychology (1973-1998). J Couns Psychol 1999; 46: 496-503.

[18] Wasserstein SB, Lopez N, Routh DK. The Journal of Abnormal Child Psychology at 25. J Abnorm Child Psychol 1997; 25: 1-5.

[19] American Psychological Association. Developmental Psychology journal description [Online]. 2008 Dec [cited 2009]. Available from: http://www.apa.org/journals/dev/description.html

[20] Feingold A. Assessment of journals in social science psychology. Am Psychol 1989; 44: 961-4.

[21] SpringerLink. Journal of Abnormal Child Psychology description [Online]. [cited 2009]. Available from: http://www.springerlink. com/content/104756/

[22] American Psychiatric Association. Diagnostic and statistical manual of mental disorders-text revision. $4^{\text {th }}$ ed. Washington, DC: American Psychiatric Association 2008.

[23] American Academy of Pediatrics. The pediatrician's role in the diagnosis and management of autistic spectrum disorder in children. Pediatrics 2001; 107: 1221-6.

[24] Malmquist CP. Depressive phenomena in children. In: Wolman BB, Ed. Manual of child psychopathology. New York: Mc-Graw Hill 1972; pp. 497-540.

[25] Costello EJ, Erkanli A, Angold A. Is there an epidemic of child or adolescent depression? J Child Psychol Psychiatry 2006; 47: 126371.

[26] Barkley RA. Attention-Deficit/Hyperactivity Disorder. In: Mash, EJ, Barkley RA, Eds. Child psychopathology. New York: Guilford 2003; pp. 75-143.

[27] Chakrabarti S, Fombonne E. Pervasive developmental disorders in preschool children: Confirmation on high prevalence. Am J Psychiatry 2005; 162: 1133-41.

[28] Jensen PS. Comorbidity and child psychopathology: Recommendations for the next decade. J Abnorm Child Psychol 2003; 3: 293-300. 